\title{
FEATURES OF THE PROCESSES OF ELASTIC DEFORMATION IN CUBIC CRYSTALS
}

\author{
E. A. STREBKOVA ${ }^{1,2^{*}}$, M. N. KRIVOSHEINA ${ }^{1,2}$ AND YA. V. MAYER ${ }^{1,2}$ \\ ${ }^{1}$ Institute of Strength Physics and Material Science of the Siberian Branch of RAS \\ Akademicheskii 2/4, 634021 Tomsk, Russia \\ ${ }^{2}$ Tomsk State University, Lenina Avenue 36, 634050 Tomsk, Russia \\ *Corresponding author. E-mail: elenatuch@yandex.ru
}

DOI: $10.20948 /$ mathmontis-2019-46-8

\begin{abstract}
Summary. The processes of elastoplastic deformation in single-crystal alloys characterized by cubic symmetry of properties are investigated. Using the heat-resistant single-crystal alloy VZhM8 used to create gas turbine engine blades by directional crystallization as an example, the dependences of deformation processes on the orientation of loading directions with respect to crystallographic axes are shown. Significant anisotropy of mechanical properties, including the presence of negative Poisson's ratios, in heat-resistant nickel alloys is maintained up to a temperature of $1150{ }^{\circ} \mathrm{C}$. Therefore, over the entire range of operating temperatures, the propagation velocities of elastic and plastic waves in single-crystal heat-resistant nickel alloys depend on the propagation direction. On the example of a VZhM8 single-crystal alloy under dynamic loading in a three-dimensional formulation, the differences in the processes of deformation realized in a single crystal under loading along the [011], [111] and [001] axes are investigated.
\end{abstract}

\section{INTRODUCTION}

The mechanical properties of anisotropic materials, which include single crystals [1] with cubic symmetry properties, depend on the direction. When they are loaded in some directions, a common feature is auxeticity (deformation of the same sign in the direction perpendicular to the direction of loading). In single crystals with cubic symmetry of properties [2-9], elastic properties in the plane (011) are traditionally subject to investigation. This is due to the presence of negative values of Poisson's coefficients (auxeticity) in some planes, as well as values exceeding 0.5 and even 1.5 for some types of single crystals. Therefore, the processes of elastic deformation under loading of single crystals along the axis [011] have a number of features. The problems of single crystal deformation with cubic symmetry of properties under dynamic loading conditions [10] are considered, for example, single-crystal VZhM8 based on nickel, with face-centered lattice. In materials with cubic symmetry of properties in the (011) plane, the elastic properties coincide only when the axes are rotated by an angle of $90^{\circ}$; for any other angles of rotation in the (011) plane, the elastic properties are different. Therefore when the shock loading direction changes relative to the crystallographic axes of single crystals with cubic symmetry

Key words and phrases: single crystals, auxeticity, shock loading, spall fracture. 
of properties, as well as when the computational coordinate system rotates in any plane relative to the crystallographic axes, the wave pattern of deformation changes. Turbine blades of heat-resistant nickel alloys are made from such materials. VZhM8 V generation single-crystal alloy, created for use by gas turbine engine blades, is characterized by significant anisotropy of elastic properties, plasticity, creep, short-term and long-term strength, high-cycle and lowcycle fatigue, and crack resistance [11]. Alloy VZhM8 contains $\mathrm{Cr}-\mathrm{vol} 3 \%$, Mo-vol3.5\%, $\mathrm{W}-\operatorname{vol} 4.2 \%, \mathrm{Re}-\operatorname{vol} 6.3 \%, \mathrm{Ta}-\operatorname{vol} 6 \%, \mathrm{Al}-\operatorname{vol} 5.7 \%, \mathrm{Co}-\operatorname{vol} 5.5 \%, \mathrm{Ru}-\operatorname{vol} 6 \%$.

The single-crystal alloy VZhM8 has a dendritic structure. It has a heterophase structure. The rhenium-containing alloy is additionally alloyed with ruthenium to stabilize the phase composition. All mechanical characteristics have their own anisotropy [12], which significantly depends on temperature. Aircraft engine blades are grown in such a way that their longitudinal axis coincides with the crystallographic direction [001]. This is determined by the need to minimize the magnitude of the Young's modulus and, consequently, the magnitude of thermal stresses in the direction of the centrifugal forces. The azimuthal orientation of the single-crystal alloy with respect to the blade geometry is not controlled [13-15]. To study the mechanical properties in the [001], [011] and [111] directions in single crystal alloys, sample castings are obtained using seed single crystals of $\mathrm{Ni}-\mathrm{W}$ alloy. The samples have a cylindrical shape; the longitudinal axis of the samples coincides with one of the directions: [001], [011] and [111]. Poisson's coefficients, Young's modulus under tensile conditions at different temperatures [16], as well as plasticity and strength characteristics are investigated in the obtained samples. The values of elastic and plastic properties of the VZhM8 alloy obtained in full-scale experiments in the directions [001], [011] and [111] in [17] were used in modeling the processes of elastic and plastic deformations.

Using the example of solving a test problem (Taylor test) with different orientations of the crystallographic axes of a single crystal with cubic symmetry properties relative to the cylinder axis in a three dimensional formulation, features of the deformation processes characteristic of auxetic materials are shown. These studies are needed to corrected the mathematical models used to process the results of field experiments used to study the dynamic properties of materials $[18,19]$. The values of the propagation velocities of elastic waves depending on the direction in anisotropic and in particular auxetic materials play an important role in such field experiments.

The paper presents the results of numerical simulation of spall fracture of a target from a VZhM8 alloy for the case of coincidence of the direction of shock loading with the [011] axis and it is shown that in this case a non-axisymmetric deformation process occurs in the target. From the solution of the problem of shock loading of the cylinder along the [011] direction the reason of this asymmetry is clear. In the study of the deformation processes for the three cases of loading of the cylinders against a rigid wall and shock loading targets carried out nu- 
merically by the dynamic finite element method using original programs [20,21]. The aim of the work is to study the processes of elastic-plastic deformation in materials characterized by cubic symmetry of mechanical properties under dynamic loads. The influence of the presence of negative Poisson's coefficients in the elastic properties of cubic single crystals on the implementation of various processes of elastic-plastic deformation depending on the directions of shock loading relative to the crystallographic axes of single crystals is shown. A mathematical model of elastic-plastic modeling of anisotropic materials is applied, taking into account the correspondence of uniform bulk deformation to anisotropic stress.

\section{MATHEMATICAL STATEMENT OF THE PROBLEM}

Dynamic loading of an anisotropic solid is modeled within the framework of continuum mechanics using the continuity equation and equations of motion [22] in a three dimensional formulation: continuity equation

$$
\frac{\mathrm{d} \rho}{\mathrm{d} t}+\rho \operatorname{div} \bar{v}=0
$$

continuum motion equations

$$
\rho \frac{\mathrm{d} v^{k}}{\mathrm{~d} t}=\frac{\partial \sigma^{k i}}{\partial x_{i}}+F^{k}
$$

where $\rho$ is the medium density; $v$ is speed vector; $F^{k}$ are the mass vector components; $\sigma^{i j}$ are the contravariant components of the symmetric stress tensor. The components of the symmetric strain rate tensor $\left(e_{i j}\right)$ were calculated as follows $e_{i j}=\left(\nabla_{i} v_{j}+\nabla_{j} v_{i}\right) / 2$, where $v_{j}$ are the velocity vector components; $i, j=x, y, z$. The elastic deformation of an anisotropic material is carried out using the values of total stresses and rates of total deformations and is described by the generalized Hook's law:

$$
\mathrm{d} \sigma_{i j} / \mathrm{d} t=C_{i j k l} e_{k l},
$$

where $C_{i j k l}$ are the components of the elastic constant tensor in the calculated coordinate system. The calculations were carried out using a mathematical model that includes the decomposition of the total stress tensor into the deviator part and the "anisotropic" hydrostatic stress [21]:

$$
\sigma_{i j}=S_{i j}-P_{e} \lambda_{i j}
$$

where $S_{i j}$ are the total stress deviator components, $\lambda_{i j}$ is the generalized Kronecker symbol, $P_{e}$ is the mean pressure. In the field of elastic deformations $S_{i j}=C_{i j k l} \varepsilon_{k l}, \lambda_{i j}=C_{i j k l} \delta_{k l} /\left(3 K_{a}\right)$, $K_{a}=C_{i j k l} \delta_{i j} \delta_{k l} / 9, P_{e}=\varepsilon_{V} C_{i j k l} \delta_{i j} \delta_{k l} / 3$, where $K_{a}$ is the generalized bulk strain modulus, $\delta_{k l}$ is the Kronecker symbol, $\varepsilon_{k l}$ are the deformation deviator components, $C_{i j k l}$ are the elastic constants defined in directions that coincide with the directions of the calculated coordinate system, $\varepsilon_{V}$ is volumetric deformation of anisotropic medium. In the field of elastic deformations 
$\varepsilon_{V}=\varepsilon_{11}+\varepsilon_{22}+\varepsilon_{33}$. In the field of plastic deformations $\varepsilon_{V}=\left(V-V_{0}\right) / V_{0}$. The volumetric deformation of the anisotropic medium does not change, when the direction of the deformation changes, but due to different compressibility factors of the material in different directions, it causes anisotropic hydrostatic stress in cases when $\lambda_{i j}$ is not equal to one.

Using of numerical calculations in the field of elastic deformations of the decomposition of the total stress tensor in the form of (4) is equivalent to calculations in full stresses. The total stresses in the field of plastic deformations were also calculated by formula (4). Those in the present work, this approach is extended to the region of plastic deformations and it was assumed that the pressure anisotropy coincided in the region of elastic and plastic deformations. When modeling plastic deformation of anisotropic material, the average pressure $P_{e}$ in the material was calculated using the Mie-Grüneisen equation as a function of specific internal energy $E$ and current density:

$$
P_{e}=\sum_{n=1}^{3} K_{n}\left(\frac{V}{V_{0}}-1\right)^{n}\left[1-K_{0} \frac{\left(V / V_{0}-1\right)}{2}\right]
$$

where $K_{0}, K_{1}, K_{2}, K_{3}$ are the material constants, $V, V_{0}$ are the current and initial volumes. In the field of plastic deformations, $P_{e}$ was also multiplied by the values of the coefficients $\lambda_{i j}$. The components of the total stress deviator were calculated using the flow theory. The associated law of flow is used to calculate the plastic deformation in the form

$$
\mathrm{d} \varepsilon_{i j}^{p}=\mathrm{d} \lambda \frac{\partial F}{\partial \sigma_{i j}}
$$

the parameter $\mathrm{d} \lambda=0$ at elastic deformation, at plastic is always positive, is defined by means of a condition of plasticity, $\mathrm{d} \varepsilon_{i j}^{p}$ are the components of plastic deformation, $F$ is the plasticity function.

The Mises-Hill plasticity condition, written through stress deviators for transtropic material with regard to isotropic hardening, has the form [23]

$$
F\left(S_{i j}, R\right)=\frac{S_{11}^{2}}{r_{1}^{2}}+\frac{S_{22}^{2}}{r_{2}^{2}}+\frac{S_{33}^{2}}{r_{3}^{2}}+\frac{S_{12}^{2}}{r_{4}^{2}}+\frac{S_{31}^{2}}{r_{5}^{2}}+\frac{S_{23}^{2}}{r_{6}^{2}}-R^{2}=0,
$$

where $r_{i}$ is determined through yield limits for tensile and shear transtropic material, $R$ is the isotropic hardening function.

From the experimental studies presented in [23] it is known that the function $R$ characterizing isotropic hardening is invariant to the type of stress state, is determined from experiments on simple loading and depends linearly on the accumulated plastic deformation $\varepsilon^{p}: R\left(\varepsilon^{p}\right)=1+$ $\xi \varepsilon^{p}$, where $\varepsilon^{p}=\int\left|\mathrm{d} \varepsilon_{k l}^{p}\right|, k, l=1,2,3$. 
The elastoplastic material deformation model is supplemented by a fracture criterion containing the ultimate porosity of the material. Spalling of the sample is considered as a process of merging micropores in a plastically deformed material under the action of tensile stresses. By averaging over the volume, we pass from the volume of the porous medium to the solid medium with the averaged density and the equation of state of the porous material.

Assuming that the change in pore volume depends on the plastic characteristics of the material and does not depend on the viscosity characteristics, an equation is solved that is the equality of the pressure increment obtained by the equation of state of the matrix material and due to the growth of the spherical pores. As a measure of damage, the porosity parameter $\alpha$ introduced by Herrmann. The porosity parameter in the Herrmann model is the ratio of the specific volume $\left(V=V_{\mathrm{m}}+V_{\mathrm{p}}\right)$ of the porous medium to the specific volume of the solid matrix material (starting material):

$$
\alpha=V / V_{\mathrm{m}}
$$

In modeling of the detachment fracture, an equation was used to determine the porosity parameter, obtained from the equilibrium condition of the spherical pore under the action of the applied pressure, in the following form:

$$
\alpha P_{e}+\alpha_{\mathrm{s}} \ln (\alpha /(\alpha-1))=0
$$

where $\alpha_{\mathrm{s}}=2 \sigma_{\mathrm{sm}} / 3$. This equation is applied provided that

$$
P_{e}<\alpha_{\mathrm{s}} \ln (\alpha /(\alpha-1))
$$

otherwise the change in porosity does not occur in time, i.e.

$$
\mathrm{d} \alpha / \mathrm{d} t=0
$$

The moment of completion of the local macroscopic destruction of the material is the achievement of porosity of the critical value. The elastic-plastic deformation of the isotropic projectile material was carried out using the Prandtl-Reiss model. The stresses defined in the element, rigidly rotated in space, are recalculated using the Jaumann derivative

$$
\frac{\mathrm{D} \sigma_{i j}}{\mathrm{D} t}=\mathrm{d} \sigma_{i j} / \mathrm{d} t-\sigma_{i k} \omega_{j k}-\sigma_{j k} \omega_{i k}
$$

where $\omega_{i j}=0.5\left(\partial v_{j} / \partial x_{i}-\partial v_{i} / \partial x_{j}\right)$. 


\section{COMPUTATIONAL EXPERIMENT}

The impact of a cylinder with a height of $50 \mathrm{~mm}$ and a radius of $5 \mathrm{~mm}$ from a single crystal alloy VZhM8 on a rigid target with an initial velocity of $50 \mathrm{~m} / \mathrm{s}$ is considered. The axis of symmetry of the cylinder in each problem coincides with one of the three axes in which the elastic, plastic, and strength properties in cubic crystals are traditionally investigated: [111], [001], and [011]. In the first case, all three calculated axes coincide with the directions [111]. In the second case, the direction of the axis of symmetry of the cylinder and the other two coincides with the direction of the crystallographic axes [001], [010] and [100]. In the third case, the axis of symmetry of the cylinder coincides with the direction [011], and the other two axes of the calculated coordinate system with [100] and [011]. Those, in the first and in the second cases, axisymmetric problems are solved, and in the third case, a three-dimensional stress state is realized in the cylinder. The differences in each case of the axes of symmetry of a single crystal alloy relative to the axis of symmetry of the cylinder leads to the fact that in all three problems the values of technical elastic constants and velocities of propagation of elastic waves in the direction of each coordinate axis have different values. Only elastic deformations are considered, in order to determine their contribution in cases of development of elastoplastic deformations. The ratios of the strain values are analyzed, as well as their sign in three cases of different orientations of the VZhM8 crystallographic axes with respect to the direction of impact.

In the first case, the values of technical elastic constants are: $E=250.6 \mathrm{GPa}, v=0.28$, $G=46.7 \mathrm{GPa}$, longitudinal wave speeds $v_{L}=6548 \mathrm{~m} / \mathrm{s}$, shear wave speeds $v_{S}=2646 \mathrm{~m} / \mathrm{s}$.

In the second case, the values of technical elastic constants are: $E=102.2 \mathrm{GPa}, v=0.426$, $G=118.7 \mathrm{GPa}$ longitudinal wave speeds $v_{L}=5539 \mathrm{~m} / \mathrm{s}$, shear wave speeds $v_{S}=3619 \mathrm{~m} / \mathrm{s}$.

In the third case, the values of technical elastic constants differ in three mutually perpendicular directions: $E_{x}=102.2 \mathrm{GPa}, E_{y}=E_{z}=193.2 \mathrm{GPa}, G_{x y}=G_{z x}=118.7 \mathrm{GPa}, G_{y z}=35.8 \mathrm{GPa}$, $v_{x y}=0.788, v_{y z}=-0.14, v_{z x}=1.489$, longitudinal wave speeds $v_{L}=6311 \mathrm{~m} / \mathrm{s}$, shear wave speeds $v_{S 1}=3619 \mathrm{~m} / \mathrm{s}$ and $v_{S 2}=1989 \mathrm{~m} / \mathrm{s}$ [13]. In this case, the cylinder material is characterized by auxeticity; the other two Poisson ratios exceed the value of 0.5 . Material density $\rho_{0}=9060 \mathrm{~kg} / \mathrm{m}^{3}$. The axis of symmetry of the cylinder in all three cases coincides with the axis $O Z$. The shock loading of a cylindrical body made of VZhM8 alloy with a steel projectile with an initial velocity of $600 \mathrm{~m} / \mathrm{s}$ is simulated. The elastoplastic deformation of the isotropic material of the projectile is determined using the Prandtl-Reuss model. Plastic deformation in the material of an target is determined using a flow model with isotropic hardening. The Mises-Hill function is used as a function of plasticity for anisotropic media. The yield strength at a temperature of $800{ }^{\circ} \mathrm{C}$ is $934 \mathrm{MPa}$ in the [011] direction and $1050.8 \mathrm{MPa}$ in the [001] direction. The spallation of a cylindrical body from a VZhM8-target alloy is modeled on the base of a mathe- 


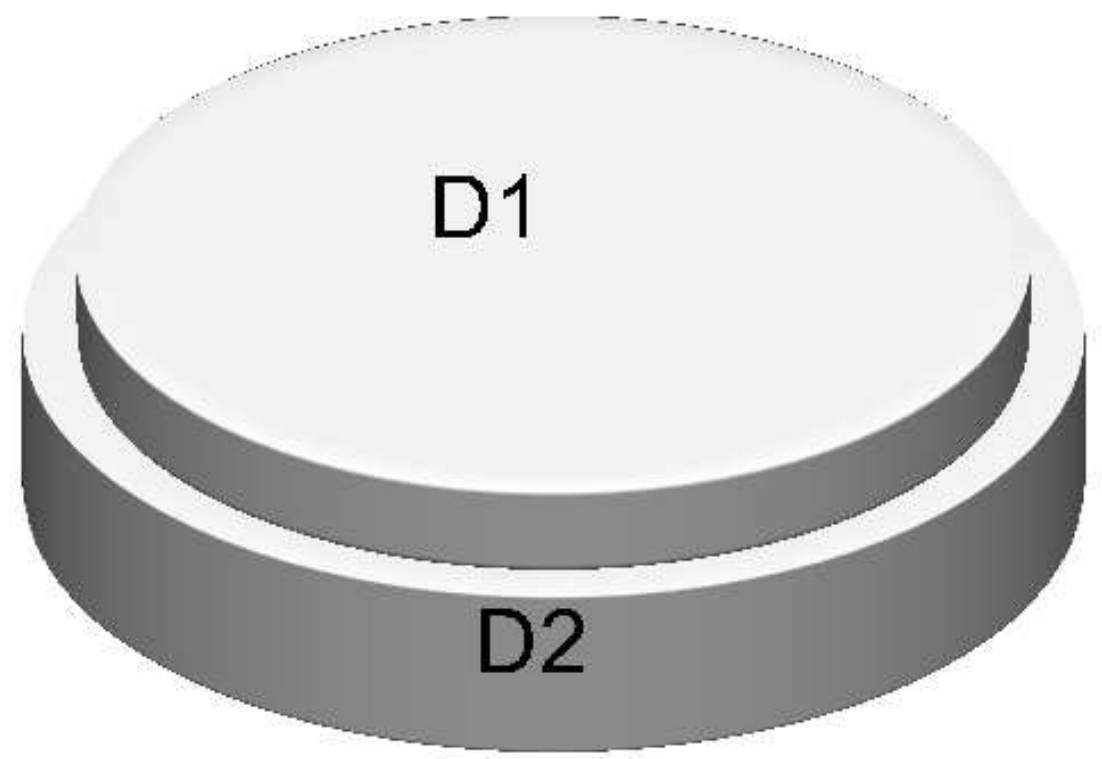

Figure 1: The initial configuration of the projectile and target.

matical model modified for anisotropic media [24]. The element is considered to be destroyed and all stress components in it are equated to zero when a local stretch of the grid element is more than $30 \%$. This simulates the appearance of a spall crack in the material of a target with its shock loading. Consider the interaction of two bodies in the general, three-dimensional case in the Cartesian coordinate system $X Y Z$ (figure 1).

The calculation method used is the finite element method modified by G. R. Johnson for solving problems in dynamic formulation [20]. It has first order accuracy in time and in space. Discretization of the computational domains was carried out using simplex approximation. Boundary conditions of impact-loading of the cylinder on a rigid wall. Each body occupies an area $D_{i}(i=1,2)$, bounded by the surface $Q_{i}$, respectively. The velocity vector of the striker at the initial moment of time has a value $V_{0}$ and its direction coincides with the longitudinal axis of the striker. Surfaces $Q_{1}$ and $Q_{2}$ are stress-free, $Q_{3}$-the contact surface between bodies. Each material is in a non-stressed undeformed homogeneous state. Initial conditions $(t=0): u=V_{0}$, $\sigma_{i j}=E=w=v=0$, with $x, y, z \in D_{1}(i=x, y, z), \sigma_{i j}=E=w=v=u=0$, with $x, y, z \in$ $D_{2}(i=x, y, z), \rho=\rho_{i}$, with $x, y, z \in D_{i}, i=1,2$, Where $u, v, w$ are the components of the velocity vector along the axes $X Y Z$ are respectively. The boundary conditions are as follows: on free 
surfaces the conditions are fulfilled $T_{n n}=T_{n s}=T_{n b}=0$, friction-free sliding conditions are realized on the contact surfaces $T_{n n+}=T_{n n-}, T_{n b+}=T_{n b-}=T_{n s+}=T_{n s-}=0, v_{n+}=v_{n-}$. Here, $n$ is the unit vector of the normal to the surface at the point under consideration, $b$ and $s$ are the unit vectors tangent to the surface at this point, $T_{n}$ is the force vector on the site with the normal $n, v$ is the velocity vector. The lower indices of the vectors $T_{n}$ and $v$ mean projections on the corresponding vectors of the basis; the plus " + " characterizes the value of parameters in the material at the upper boundary of the contact surface, the minus "-" - at the bottom.

Discretization of computational domains. To construct a uniform numerical grid of nodes in the computational domain, an algorithm for constructing a grid in a Cartesian coordinate system is used [25]. Simplex elements-tetrahedra-were used to construct the grid in a threedimensional coordinate system. The mass of the element was evenly distributed between the four nodes. In cases where a node belonged to several elements, the total mass, concentrated in the $i$-th node was equal to one-fourth of the mass of all elements containing this node.

\section{THE DISCUSSION OF THE RESULTS}

Figure 2 shows a decrease in the length of the cylinders, for three cases of orientation of the crystallographic axes relative to the axis of symmetry of the cylinder. Curve 1 shows that due to the maximum values of the elastic properties along the [111] direction, the oscillations of the cylinder length have a shorter period. For the considered ratio of the length of the cylinder to its diameter, the oscillations of the length of the cylinder are determined by the values of the rod ones, not the longitudinal speeds. Figure 2 shows that there is a clear relationship between the periods of oscillations of the lengths of the cylinders and the values of the velocities of propagation of longitudinal waves. In the case of orientation along the axis of symmetry of the cylinder, the direction of the [001] crystal changes the cylinder length to the maximum and have a maximum oscillation period (curve 2, figure 2). In all three cases, the times of arrival of the wave to the free surface of the cylinder are different due to differences in the velocities of propagation of longitudinal waves. In the case of orientation along the axis of symmetry of the cylinder, the direction of the crystal [011] (curve 3, figure 2) exceeds the maximum change in the length of the cylinder by $17 \%$ in the case of the direction [111] along the axis of the cylinder.

Auxeticity of anisotropic materials is manifested in directions perpendicular to the direction of loading. In figure 3, curve 3 shows the elastic variation of the cylinder radius along the $O Y$ axis at a height of $1 \mathrm{~mm}$ from the contact surface of the cylinder for the case of direction [011] along the axis of the cylinder with time. Until the cylinder is separated from the target, elastic oscillations of the magnitude of the cylinder radius demonstrate compressive deformation. After the cylinder is separated from the target, changes in the radius value occur around the original value. When the directions [111] or [001] coincide, the magnitudes of changes in the radiuses of 


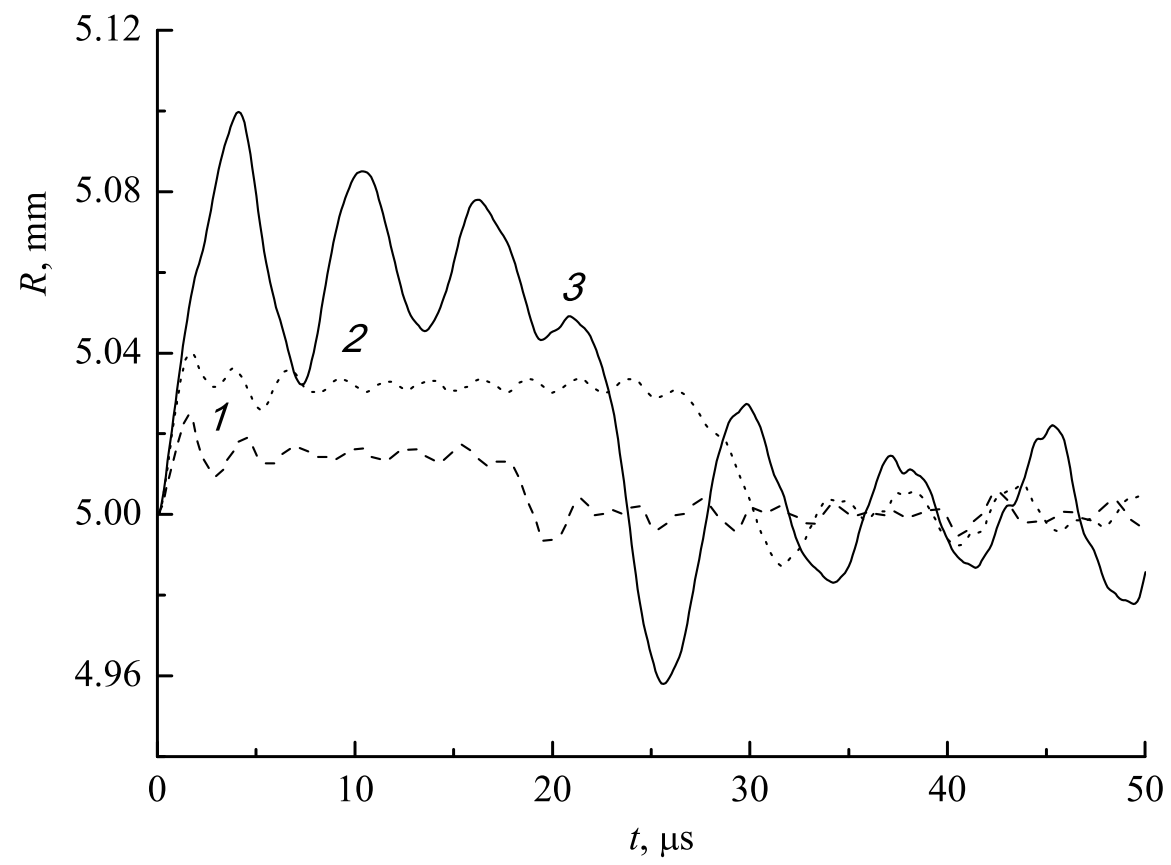

Figure 2: The change of the cylinder radius in time along the axis $O X$, for the cases: 1 - the axis of symmetry of the cylinder is directed along the [111] axis; 2-along the [001] axis; 3-along the [011] axis.

the cylinders at a height of $1 \mathrm{~mm}$ from the contact surface demonstrate only tensile deformation up to cylinder bounce. The periods and amplitudes of oscillations of the radiuses of the cylinders in these cases are much smaller. The presence of a negative value of the Poisson coefficient in the plane formed by the loading direction and the axis $O Y$ manifests itself in the compression of the cylinder along the axis $O Y$, but only during the contact time of the cylinder and target.

The elastic change in the cylinder radius is also at a height of $1 \mathrm{~mm}$ from the contact surface of the cylinder, but in the perpendicular direction, shown in figure 4 . Curve 3 shows the change in the cylinder radius over time, which significantly exceeds the change in the radiuses of the cylinders for the cases of curves 1 and 2 . In the plane formed by the direction of loading and the axis $O X$ for the material oriented in the cylinder along the direction [011] the Poisson's ratio is greater than 0.5 , i.e. exceeds the maximum value for isotropic materials. Therefore up to a cylinder bounce from an target in the case of [011] along the $O X$ axis, an increase in radius 2 times greater than in the case of orientation along the cylinder axis of the single crystal [001] is observed, with Poisson's ratio equal to 0.426 (curve 2, figure 4).

Such a ratio of changes in the radiuses of the cylinders for three cases of orientation of the crystallographic axes relative to the axes of symmetry of the cylinders is observed at any distance from the contact to the free surfaces of the cylinders. The obtained results explain the reason why a non-symmetric deformed state is realized in the target from single crystal 


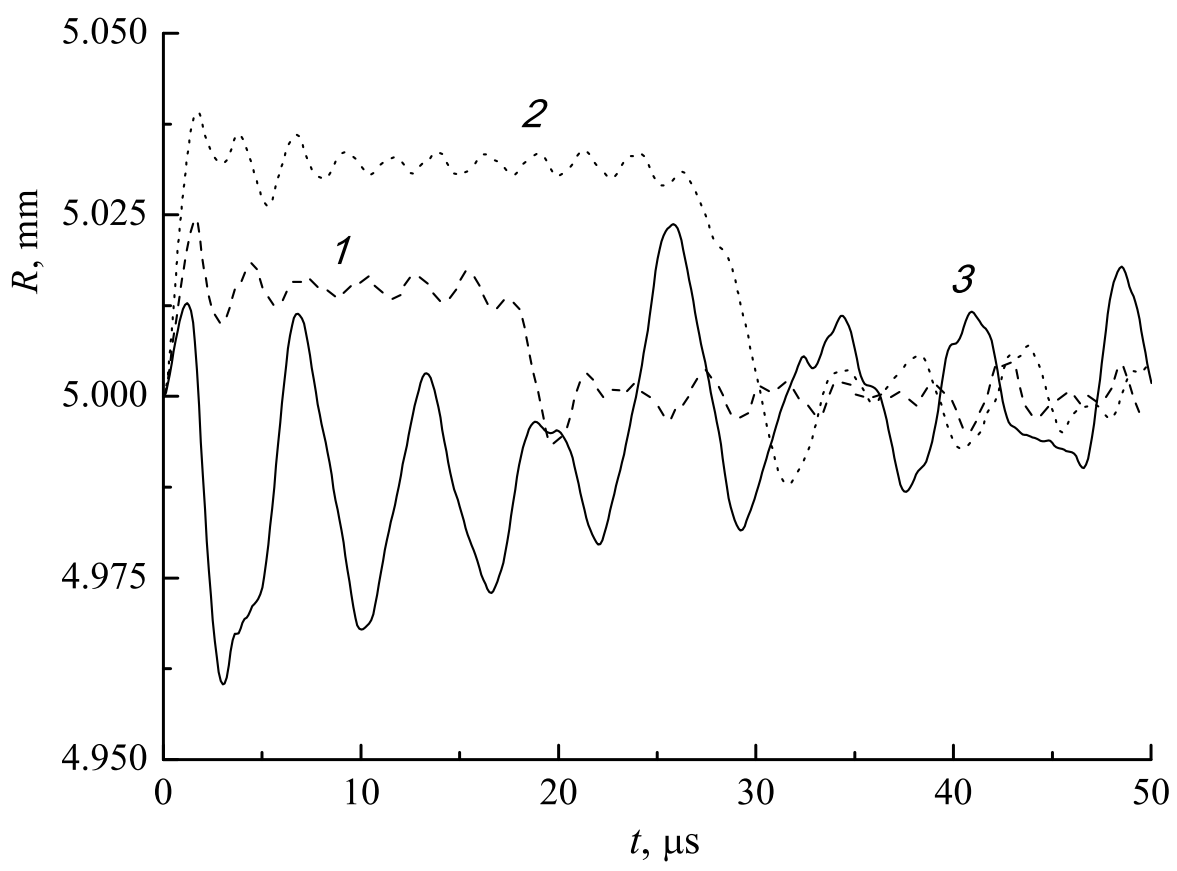

Figure 3: The change in the cylinder radius over time along the $O Y$ axis, for the cases: 1 - the axis of symmetry of the cylinder is directed along the [111] axis; 2-along the [001] axis; 3-along the [011] axis.

alloy VZhM8 with its shock loading in the direction [011]. Target from single crystal alloy VZhM8 has a cylindrical shape but its height is $2 \mathrm{~mm}$ and a radius of $7.5 \mathrm{~mm}$. Its shock loading was carried out by a steel projectile with a height of $1 \mathrm{~mm}$ and a radius of $7.45 \mathrm{~mm}$ with an initial speed of $600 \mathrm{~m} / \mathrm{s}$. In this case a problem is numerically modeled in a three-dimensional statement. It is realized in field experiments in the study of dynamic characteristics. The process of deformation in the target includes elastic, plastic deformation as well as spall fracture. The degree of deviation from axisymmetric deformation in an target can be illustrated by changing the magnitude of the target radii on the lateral surfaces along the axis $O X$ and $O Y$ (figure 5).

Since the yield strength of the VZhM8 alloy in the direction of the $O Y$ axis is less than in the direction of the $O X$ axis it was logical to expect that a larger increase in radius would be observed in the direction of the $O Y$ axis. The figure shows that by the time point of $1 \mu \mathrm{s}$, when the spalling destruction has already occurred, the target radius in its middle part along the $O X$ axis increased by $0.19 \mathrm{~mm}$, and in the $O Y$ axis direction-only by $0.13 \mathrm{~mm}$. As shown in the analysis of the process of elastic deformation under shock loading of the cylinder along the same direction [011], the missing part of the deformation in the direction of the $O Y$ axis is determined by the auxetism of the VZhM8. That is the missing part of the deformation in the direction of the $O Y$ axis is determined by the compressive elastic deformation due to the negative value of the Poisson's ratio. This calculation showed that in the study of the dynamic 


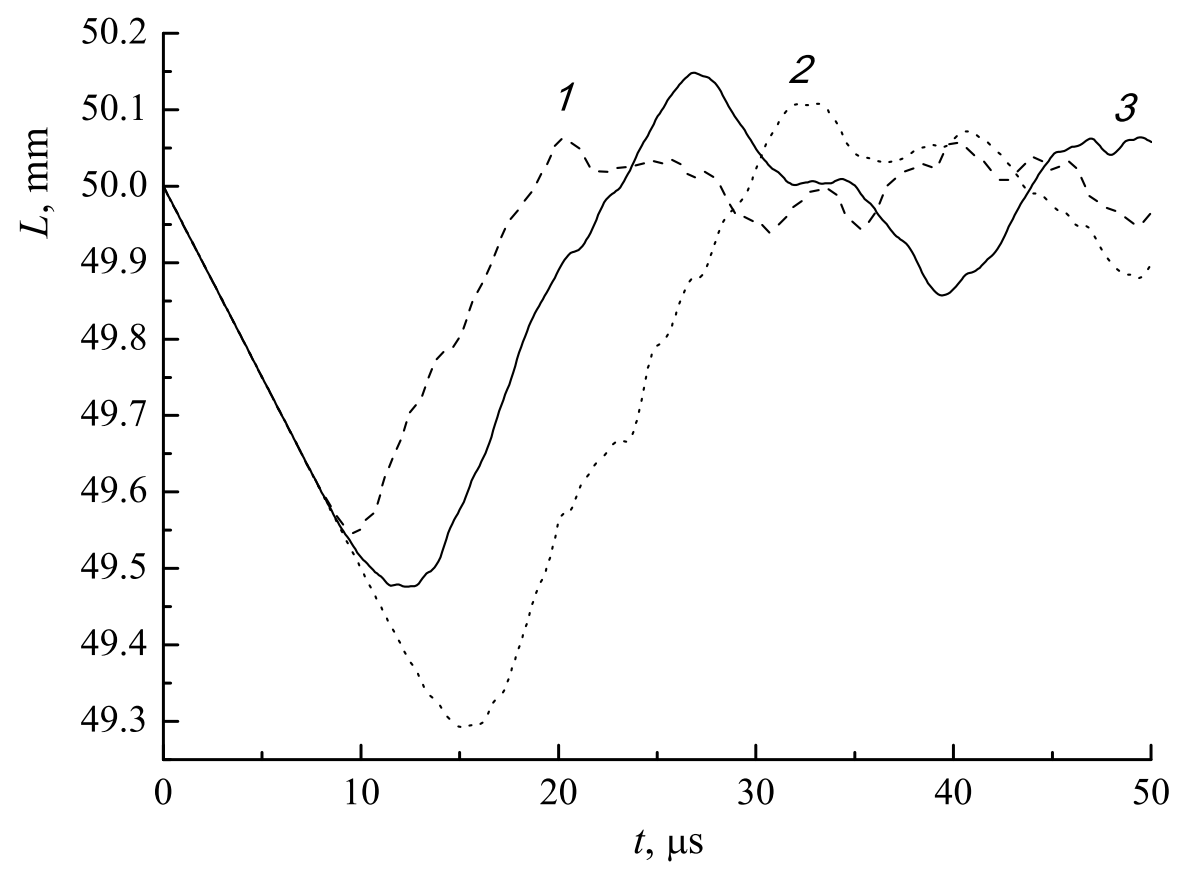

Figure 4: The change in the height of the cylinder, for the cases: 1 - the axis of symmetry of the cylinder is directed along the [111] axis; 2-along the [001] axis; 3-along the [011] axis.

properties of auxetic material with an initial shock loading speed of $600 \mathrm{~m} / \mathrm{s}$ until the moment of spall fracture the elastic properties to a greater extent determine the process of elastoplastic deformation of the target. The type and location of the spall crack in the target is shown in figure 6 .

The distribution of relative volumes $\left(V_{\mathrm{v}}=V / V_{0}\right)$ of elements is shown (the ratio of the current volume of the element of the computational grid to the initial one) in the section of the target. The figure shows that in the area where the crack was formed the volume of elements on average increased 1.5-1.75 times. The cross section of the projectile and target is made in the $O Z Y$ plane: the $O Z$ axis is directed upwards, the loading was simulated along it; $O Y$ axis is perpendicular to it. Dark blue targets areas are areas where is no stretch. In areas from blue to red there are stretch areas, where the relative volume is greater than 1 . 


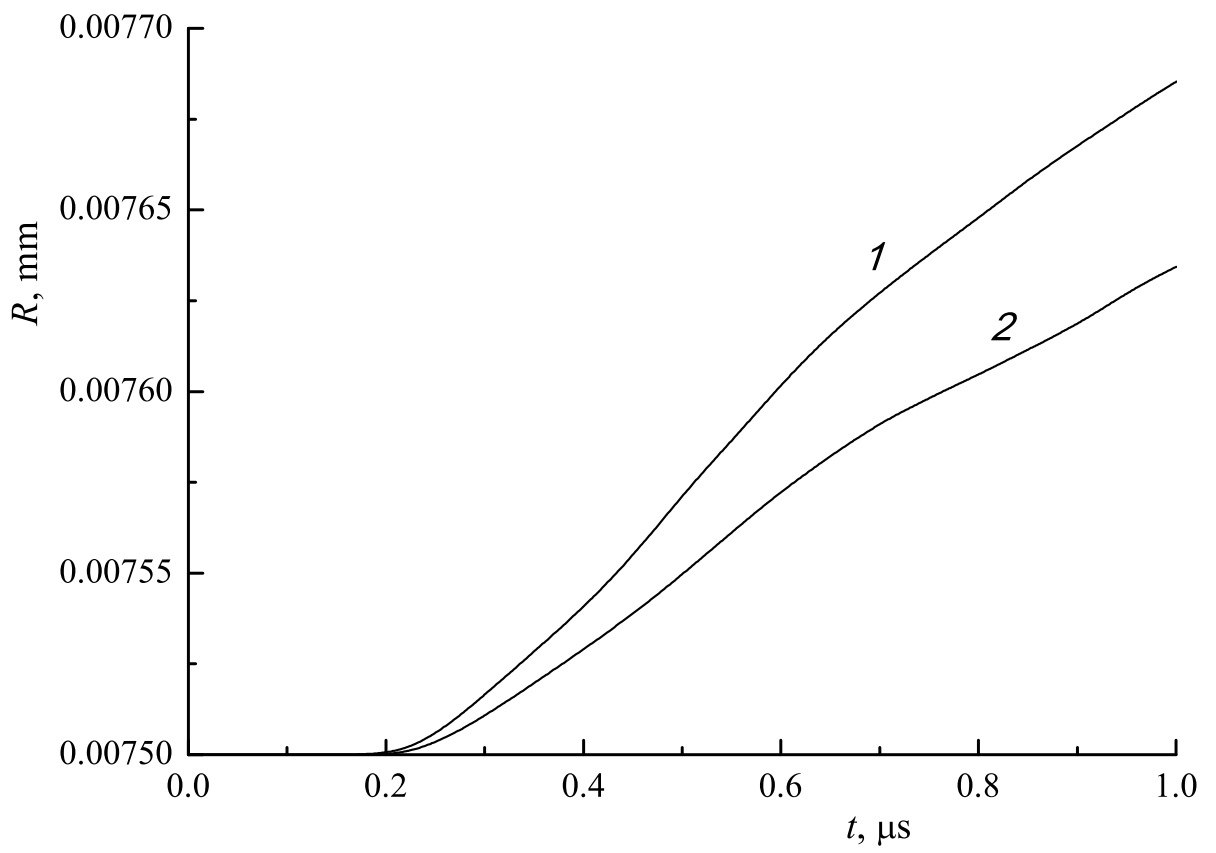

Figure 5: The change in the radius of the target in time: 1 -along $O X$ axis; 2 -along $O Y$ axis.
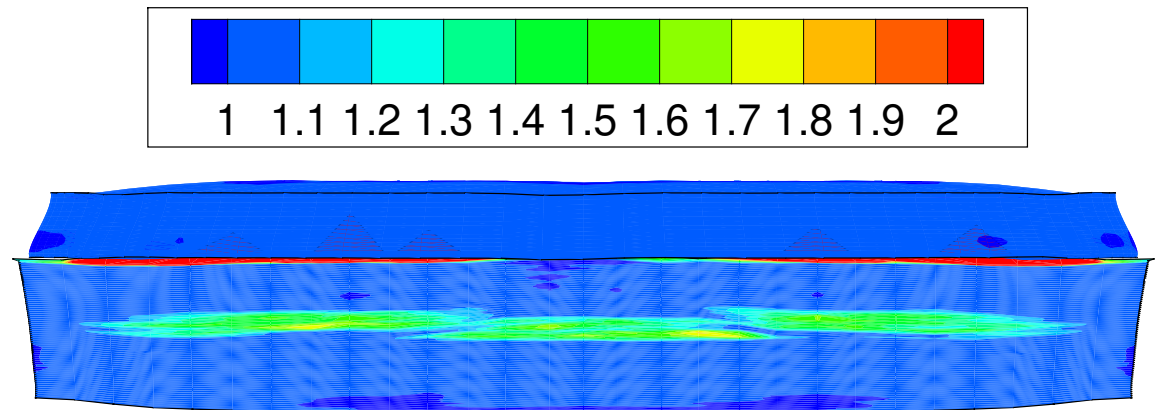

Figure 6: Distribution of the relative volume of the material in the cross section $O Z Y$ of the projectile and target at the moment $0.85 \mu \mathrm{s}$. 


\section{CONCLUSIONS}

On the example of a heat-resistant single-crystal alloy VZhM8, characterized by a significant anisotropy of mechanical properties, including auxeticity, significant differences in the processes of elastic-plastic deformation under loads along the axis [011], [111] and [001] are shown. To demonstrate the features of the elastic deformation processes for the VZhM8 alloy, the solutions of the Taylor test (cylinder impact on a non-deformable wall) obtained numerically in a three-dimensional formulation are shown. When modeling the shock loading of a thin cylindrical target made of a single-crystal alloy VZhM8 along the direction [011], it is shown that a three-dimensional elastic-plastic deformation is realized in the target, due to the auxeticity of the target material.

Acknowledgments: The work was performed in the framework of the project of the Russian Science Foundation No. 18-71-00062.

The paper is based on the proceedings of the XXXIV International Conference on Interaction of Intense Energy Fluxes with Matter, Elbrus, the Kabardino-Balkar Republic of the Russian Federation, March 1 to 6, 2019.

\section{REFERENCES}

[1] O. N. Koroleva, A. V. Mazhukin, and V. I. Mazhukin, "Modeling of silicon characteristics in the semiconductor-metal phase transition region", Math. Montis., 41, 73-90 (2018).

[2] T. C. T. Ting and D. M. Barnett, "Negative Poisson's ratios in anisotropic linear elastic media", $J$. Appl. Mech., 72, 929-931 (2005).

[3] T. P. Hughes, A. Marmier, and K. E. Evans, "Auxetic frameworks inspired by cubic crystals", Int. J. Solids Struct., 47, 1469-1476 (2010).

[4] G. N. Greaves, A. L. Greer, R. S. Lakes, and T. Rouxel, "Poisson's ratio and modern materials", Nature Materials, 10, 823-837 (2011).

[5] A.C. Branka, D. M. Heyes, and K. W. Wojciechowski, "Auxeticity of cubic materials under pressure", Phys. Stat. Sol. B, 248, 96-104 (2011).

[6] R. V. Goldstein, V. A. Gorodtsov, and D. S. Lisovenko, "Classification of cubic auxetics", Phys. Stat. Sol. B, 250, 2038-2043 (2013).

[7] U. Scharer and P. Wachter, "Negative elastic constants in intermediate valent SmLaS", Sol. St. Commun., 96, 497-501 (1995).

[8] U. Scharer and P. Wachter, "Brillouin spectroscopy with surface acoustic waves on intermediate valent, doped SmS", Phys. B, 244, 148-153 (1998).

[9] K. E. Evans, M. A. Nkansah, I. J. Hutchinson, and S. C. Rogers, "Molecular network design", Nature, 353, 124-125 (1991).

[10] K. K. Maevskii, "Thermodynamic parameters of mixtures with silicon nitride under shock-wave loading", Math. Montis., 45, 52-59 (2019).

[11] A. I. Epishin and D.S. Lisovenco, "Ekstremal'nye znacheniya koefficienta Puassona kubicheskih kristallov", Tech. Phys., 86, 74-82 (2016).

[12] S. V. Ershov, A. A. Garbul, S. G. Pozdnyakov, V. G. Sokolov, and A. G. Voloboy, "Lighting simulation in anisitropic media", Math. Montis., 39, 42-56 (2017).

[13] A.E. Solovyov, S. A. Golinec, and K. K. Khvatskiy, "Anizotropiya harakteristik uprugosti pri rastyazhenii monokristallicheskih zharoprochnyh nikelevyh splavov", Trudi VIAM, 10, 112-118 (2017). 
[14] N. Djordjevic, R. Vignjevic, N. Djordjevic, R. Vignjevic, L. Kiely, S. Case, T. Vuyst, J. Campbell, and K. Hughes, "Modelling of shock waves in fcc and bcc metals using a combined continuum and dislocation kinetic approach", Int. J. Plast., 105, 211-224 (2018).

[15] E. A. Rodas and R. W. Neu, "Crystal viscoplasticity model for the creep-fatigue interactions in single-crystal ni-base superalloy CMSX-8", Int. J. Plast., 100, 14-33 (2018).

[16] N. V. Petrushin, O. G. Ospennikova, and E. S. Elutin, "Renij v monokristallicheskih zharoprochnyh nikelevyh splavah dlya lopatok gazoturbinnyh dvigatelej", Aerospace Engineering and Technology, 5, 5-16 (2014).

[17] R. M. Nazarkin, V. G. Kolodochkina, O. G. Ospennikova, and M. R. Orlov, "Izmeneniya mikrostruktury monokristallov zharoprochnyh nikelevyh splavov v processe dlitel'noj ekspluatacii turbinnyh lopatok", Aerospace Engineering and Technology, 4, 9-17 (2016).

[18] K. V. Khishchenko, "Equation of state for magnesium hydride under conditions of shock loading", Math. Montis., 43, 70-77 (2018).

[19] K. V. Khishchenko, "Equation of state of sodium for modeling of shock-wave processes at high pressures", Math. Montis., 40, 140-147 (2017).

[20] C.E. Anderson, P. A. Cox, G. R. Johnson, and P.J. Maudlin, "A constitutive formulation for anisotropic materials suitable for wave propagation computer programs", Comput. Mech., 15, 201223 (1994).

[21] M. N. Krivosheina, S. V. Kobenko, E. V. Tuch, O. A. Kashin, A. I. Lotkov, and Yu. A. Khon, "Fracture features of anisotropic materials at different impact velocities", Eur. J. Comput. Mech., 26, 609-621 (2017).

[22] L. I. Sedov, Mekhanika Sploshnoj Sredy, Moscow: Nauka, (1976).

[23] V. V. Kosarchuk, B. I. Kovalchuk, and A. A. Lebedev, "Report 1. Opredelyayushchie sootnosheniya”, Problemy prochnosti, 4, 50-56 (1986).

[24] J. N. Johnson, "Calculation of plane wave propagation in anisotropic elastic plastic solids", J. Appl. Phys., 43, 2074 (1972).

[25] M. N. Krivosheina, A. V. Radchenko, and S. V. Kobenko, "Razrushenie ortotropnogo i izotropnogo sfericheskih tel pod dejstviem impul'sa vsestoronnego szhatiya", Mekhanika kompozicionnyh materialov $i$ konstrukcij, 7, 95-102 (2001).

Received October 21, 2019 